\title{
On the Formation of Massive Primordial Stars
}

\author{
Kazuyuki Omukai ${ }^{1,2}$ and Francesco Palla ${ }^{1}$ \\ omukai@th.nao.ac.jp, palla@arcetri.astro.it
}

\begin{abstract}
We investigate the formation by accretion of massive primordial protostars in the range 10 to $300 M_{\odot}$. The high accretion rate used in the models $(\dot{M}=4.4 \times$ $10^{-3} M_{\odot} \mathrm{yr}^{-1}$ ) causes the structure and evolution to differ significantly from those of both present-day protostars and primordial zero-age main sequence stars. After an initial expansion of the radius (for $M_{*} \lesssim 12 M_{\odot}$ ), the protostar undergoes an extended phase of contraction (up to $M_{*} \simeq 60 M_{\odot}$ ). The stellar surface is not visible throughout most of the main accretion phase, since a photosphere is formed in the infalling envelope. Also, significant nuclear burning does not take place until a protostellar mass of about $80 M_{\odot}$. As the interior luminosity approaches the Eddington luminosity, the protostellar radius rapidly expands, reaching a maximum around $100 M_{\odot}$. Changes in the ionization of the surface layers induce a secondary phase of contraction, followed by a final swelling due to radiation pressure when the stellar mass reaches about $300 M_{\odot}$. This expansion is likely to signal the end of the main accretion phase, thus setting an upper limit to the protostellar mass formed in these conditions.
\end{abstract}

Subject headings: cosmology: theory — early universe — stars: formation — stars: pre-main-sequence

\section{Introduction}

Numerical simulations are now beginning to constrain the properties of the sites where star formation first occurred in the post-recombination epoch (e.g., Abel, Bryan, \& Norman 2000; Bromm, Coppi, \& Larson 1999, 2001; Tsuribe 2001). These works indicate that the fragment mass scale of primordial gas clouds is relatively large $\left(M_{f} \sim 10^{3} M_{\odot}\right)$. On the other hand, the mass of protostellar cores formed inside these fragments is less than $10^{-2} M_{\odot}$ at the beginning of the main accretion phase (Palla, Salpeter, \& Stahler 1983; Omukai \& Nishi 1998). Because of the large reservoir, the cores continue to grow in mass owing to accretion of ambient matter. Eventually, they become ordinary stars that shine by nuclear burning. The fundamental questions are then:

\footnotetext{
${ }^{1}$ Osservatorio Astrofisico di Arcetri, Largo E. Fermi 5, 50125 Firenze, Italy

${ }^{2}$ Division of Theoretical Astrophysics, National Astronomical Observatory, Mitaka, Tokyo 181-8588, Japan
} 
What fraction of the cloud mass can be incorporated into a star? Is there an upper limit to the mass of the primordial stars forming under such circumstances?

In the context of present-day star formation, it is generally believed that stellar activity (radiation force, the expansion of an HII region, stellar winds, etc.) of sufficiently massive protostars halts accretion, thereby determining the final stellar mass (e.g., Larson \& Starrfield 1971; Nakano, Hasegawa, \& Norman 1995). Of these processes, radiation force on dust grains appears to be the most efficient, making spherical accretion above a mass of $\sim 15 M_{\odot}$ increasingly difficult for solartype metallicities (e.g., Wolfire \& Cassinelli 1987), although non-spherical accretion may enhance the maximum stellar mass (Nakano 1989).

The main differences between primordial and Pop I accreting protostars are the following. First, the opacity of the infalling gas is drastically reduced due to the lack of dust grains. Second, the mass accretion rate, $\dot{M}$, which is related to the sound speed $c_{\mathrm{s}}$ in the protostellar cloud by $\dot{M}=c_{\mathrm{s}}^{3} / G$

(e.g., Stahler, Shu \& Taam 1980), is substantially higher, because of the high temperature of the primordial clouds, typically $T_{g} \sim 1000-1500 \mathrm{~K}$ over a wide density range (Palla et al. 1983). These two properties imply a reduced effect of radiation pressure of the protostellar photons and a higher momentum of the inflow. Thus, reversal of accretion by radiation force alone seems to be less efficient, and a higher maximum stellar mass is expected (e.g., Larson \& Starrfield 1971). Although qualitatively correct, this prediction has not been substantiated by numerical calculations covering a large mass interval. We still do not know the sequence of events that lead to the termination of the infall at the onset of protostellar surface activity. It is the purpose of this Letter to illustrate the results of such calculations for accreting protostars in the mass range $10 M_{\odot}$ to $\sim 300 M_{\odot}$.

\section{Numerical Approach}

The evolution of low- and intermediate-mass primordial protostars has been followed by Stahler, Palla, \& Salpeter (1986, hereafter SPS). In that study, an initially small core of $0.01 M_{\odot}$ grows rapidly by accretion at a rate of $\dot{M}=4.4 \times 10^{-3} \mathrm{M}_{\odot} \mathrm{yr}^{-1}$, corresponding to the ambient gas temperature. As shown by Omukai \& Nishi (1998), this is consistent with the physical conditions in cloud fragments at the time of core formation. The calculation of SPS was terminated at a mass $M_{*}=10.5 \mathrm{M}_{\odot}$, when nuclear burning had not yet started and the core was well below the mass scale of the parent cloud. At that point, the protostar was undergoing an internal readjustment due to strong heat transfer, resulting in the appearance of a luminosity wave at the surface and radius swelling.

The basic strategy and equations used here are the same as in SPS. In this scheme, the protostellar evolution is treated as a sequence of steady state accretion flows onto a growing hydrostatic core (see Fig.1 of SPS for a definition of the various regions). The core is assumed to be in hydrostatic equilibrium and the ordinary stellar structure equations are applied. If the gas in front of the accretion shock is optically thin, no envelope model is constructed and the boundary condition 
eq. (4a) of SPS is applied at the core surface. In case the preshock gas is optically thick, we integrate the equations inside the radiative precursor from the photosphere to the core surface (see eqs. 7a-d of SPS). Outside the photosphere, we assume a free-falling flow and evaluate at each time the deceleration exerted by radiation pressure. We adopted the same mass accretion rate of $\dot{M}=4.4 \times 10^{-3} M_{\odot} \mathrm{yr}^{-1}$ as in SPS. The main difference with that work is in the use of updated opacity tables: for $T<7000 \mathrm{~K}$, we take the results of Lenzuni, Chernoff, \& Salpeter (1991, composition of $X=72, Y=28$ ), and the OPAL opacity at higher temperatures (Iglesias \& Rogers 1996, with slightly different composition $X=70, Y=30)$.

\section{Results}

The evolution of primordial protostars in the range $0.01 M_{\odot}$ to $10.5 M_{\odot}$ has been computed by SPS. According to their results, early in the evolution, the accreted material settles adiabatically. Because of the high value of $\dot{M}$, the core is surrounded by an optically thick radiative precursor. As the protostar grows, the luminosity increases until the Kelvin-Helmholtz (KH) time scale becomes shorter than the accretion time. The contraction causes the outward propagation of the accumulated heat as a luminosity wave, which reaches the surface at a core mass of $M_{*} \sim 8 M_{\odot}$. As a result, the radius starts increasing, and the radiative precursor disappears. Owing to the complexity of the internal readjustment caused by the luminosity wave, we decided to follow again this phase and chose the $M_{*} \simeq 8 M_{\odot}$ protostar of SPS as the initial model.

\subsection{Overall evolution}

The evolution of the core $\left(R_{*}\right)$ and photospheric $\left(R_{\mathrm{ph}}\right)$ radii is presented in Figure 1. Initially, the expansion of the core continues from $M_{*}=8$ to $11.5 M_{\odot}$, reaching a maximum value of $220 R_{\odot}$. Then, due to the very short KH time, the protostar starts contracting, and the radiative precursor appears again at a mass of $M_{*}=12.4 M_{\odot}$. Therefore, except for the short interval between $M_{*} \sim 8$ and $12 M_{\odot}$, the optically thick precursor persists throughout the main accretion phase. Unlike the core radius, the photospheric radius steadily expands as the photospheric luminosity $\left(L_{\mathrm{ph}}\right)$ increases (see Fig. 2). The spatial extent of the precursor roughly corresponds to that of the ionized region because the photospheric temperature, $T_{\text {eff }} \simeq 6000 \mathrm{~K}$, is close to the ionization

temperature. However, the present case is different from an ordinary HII region in that here the gas is optically thick to electron scattering and $\mathrm{H}^{-}$bound-free absorption, in addition to Lyman continuum.

The evolution of the interior $\left(L_{*}\right)$ and photospheric luminosities divided by the core mass is shown in Figure 2. The photospheric luminosity is the sum of $L_{*}$ and $L_{\text {acc }}$, the accretion luminosity. As the luminosity-to-mass ratio $L / M_{*}$ grows, the effect of radiation force increases relative to that of gravity. Also, the accretion luminosity $L_{\mathrm{acc}} \simeq G M_{*} \dot{M} / R_{*}$ increases as a result of core contraction. 
Because of this effect and the high accretion rate, $L_{\mathrm{ph}}$ approaches the Eddington limit $\left(L_{\mathrm{Edd}}\right)$ at $M_{*} \sim 60 M_{\odot}$. At this point, the core radius has shrunk to $R_{*} \sim 12 R_{\odot}$, a value which is still higher than that of a zero-age main sequence star $\left(2.8 R_{\odot}\right.$ for a $50 M_{\odot}$ star, Marigo et al. 2001).

Despite the large increase of the interior luminosity, accretion is not halted by the effect of radiation pressure. What happens instead is a second phase of expansion of the surface regions of the core starting at $M_{*} \sim 60 M_{\odot}$ (see Fig. 1) and a concurrent reduction of the accretion luminosity. The reason for the rapid increase of the radius is the following. While the inner part of the core continues contracting (see the curve of $R_{*}(90 \%)$ in Fig. 1), the interior luminosity $L_{*}$ rises roughly as $M_{*}^{2}$ and becomes close to the Eddington limit at $M_{*} \sim 80 M_{\odot}$. At this time, hydrogen burning begins and core contraction stops. Owing to the high interior luminosity, strong radiation forces act on the thin surface layers, where the opacity sharply peaks due to the ionization of hydrogen and helium. This causes an acceleration of the expansion of the core from $\sim 80 M_{\odot}$ to $\sim 100 M_{\odot}$. Then, the ratio $L_{*} / M_{*}$ levels off at a value close to, but still smaller than, the Eddington limit (see Fig. 2) and the radius stops increasing.

From then on, the surface layers of more massive protostars are subject to a delicate balance between the radiation force and the external pressure of the accreting envelope, whereas the interior relaxes toward the structure appropriate to a main sequence star (see the evolution of $R_{*}(90 \%)$ in Fig. 1). The interior luminosity is always close to the Eddington limit and then increases approximately as $M_{*}$, although the ratio $L_{*} / M_{*}$ keeps increasing slowly. After further shrinking of the core (between 100 and $260 M_{\odot}$ ), the photospheric luminosity eventually reaches the Eddington

limit. The physical conditions in the surface layers are thus similar to those that occurred at the time of the first expansion, but now the course of events is much more dramatic. The interior luminosity $L_{*}$ continues to increase and eventually equals the Eddington limit at $\sim 300 M_{\odot}$. This causes the huge expansion of the outermost layers of the protostar shown in Fig. 1, while the interior does not contract anymore. The core runaway signals the end of the accretion phase.

\subsection{Evolution of the Core Interior}

At the beginning of the calculations, the temperature is highest off-center (around $q \equiv M / M_{*} \sim$ 0.1 ) and the innermost part of the core remains adiabatic. In addition to this thermally inactive part, the core consists of a radiative region and a superadiabatic surface layer. The newly accreted material is first incorporated into the superadiabatic layer, where the material is heated by radiation. In the radiative relaxation layer, the material loses entropy and contracts.

Because of the off-center temperature maximum, deuterium burning starts off-center at about $12 M_{\odot}$. The deuterium luminosity reaches a maximum between 12 and $16 M_{\odot}$, but the contribution to the total luminosity is always negligible $(<10 \%)$. Therefore, unlike Population I and II protostars, in primordial objects deuterium burning does not play an important role. The central inert part persists until the stellar mass reaches $\sim 40 M_{\odot}$, when radiative heating from outside becomes strong 
enough and the off-center temperature maximum disappears. We can see the corresponding sudden rise (drop) of the central temperature (density) in Figure 3b. Thereafter, the central temperature continues to rise gradually due to $\mathrm{KH}$ contraction.

When the core mass reaches $\sim 80 M_{\odot}$, the central temperature exceeds $10^{8} \mathrm{~K}$, needed to ignite the $3 \alpha$ reactions. Then, enough carbon is built up to drive the CN cycle and significant hydrogen burning ensues (see the sharp rise of $L_{\text {nuc }}$ in Figure 2). The onset and spread of central convection is shown in Figure 3a. Although carbon continues to be synthesized at the center, the growth of the convective core and mixing limits its abundance to values $\sim 10^{-9}$ (see Figure $3 \mathrm{~b}$ ).

Before the onset of the CN-cycle, the luminosity is generated mostly by contraction, with a minor contribution of H-burning via the pp-chain. This situation differs from the standard ZAMS models studied by several groups who find that the CN-cycle dominates the energy budget above $\sim 30 M_{\odot}$ (e.g. Ezer \& Cameron 1971). Since the high entropy of the accreted matter prevents contraction, the critical mass for the $\mathrm{CN}$-cycle is much higher in the protostellar case.

The radiation flow is absorbed by the high opacity in the superadiabatic layer. Because of the accumulated radiation energy in these regions, radiation pressure exceeds gas pressure at $M_{*} \sim 60 M_{\odot}$. The radiation dominated region quickly expands as the core mass increases. On the other hand, in the central convective core, the entropy increases in time due to nuclear burning. As a result, the central temperature remains almost constant, whereas the density decreases gradually (see Figure 3b). The ratio of radiation to total (gas+radiation) pressure keeps increasing, and eventually the central part becomes dominated by radiation at $M_{*}=145 M_{\odot}$. This region expands rapidly and merges with the outer radiation dominated region at $180 M_{\odot}$. In more massive objects, the internal structure is fully determined by radiation pressure.

\section{Discussion}

As a result of the high value of the mass accretion rate characteristic of primordial gas clouds, the structural properties of massive protostars have several unique and unexpected features. The evolutionary tracks of the photosphere and core surface in the HR diagram are displayed in Figure 4. The locus of the population III ZAMS stars computed from static models is also shown (Marigo et al. 2001; Bromm et al. 2001). The effective temperature of the protostellar core is computed from the core radius and preshock luminosity. The striking feature of the photospheric track is the almost constant value of the effective temperature. The photosphere forms in the accreting envelope, and persists almost throughout the main accretion phase. Because of the sensitivity of the $\mathrm{H}^{-}$bound-free opacity, the temperature is locked at the value of $T_{\text {eff }} \simeq 6000 \mathrm{~K}$ (see eq. $23 \mathrm{~b}$ of SPS). Observationally, primordial protostars of vastly different luminosities should have the same optical colors. On the other hand, the core surface will remain unobservable from outside because of the presence of the optically thick precursor.

From Fig. 4 we see that the track of the core surface cuts through the ZAMS track of the core 
surface at a mass $\sim 40 M_{\odot}$. However, the curve does not join the ZAMS smoothly, but follows a more elaborate path due to the behavior of the mass-radius relation. Therefore, realistic ZAMS models of Pop III stars should start with initial conditions that differ from those usually adopted on the basis of thermal equilibrium.

Also, the nuclear properties derived from the protostellar phase are quite distinctive. Deuterium burning and the p-p chain reactions have little effect on the evolution. Hydrogen burning due to the CN-cycle begins only at $\sim 80 M_{\odot}$. At this mass, the interior luminosity becomes close to the Eddington limit, so that the surface regions are subject to strong radiation forces. These conditions are favorable to the onset of activity in the form of mass loss. It is clear that continuum radiation driven winds can occur in Pop III protostars. According to the mass-radius relation of Fig. 1, there are two critical stages when the conditions become favorable for the onset of a wind: the rapid expansion of the surface layers at $M_{*} \sim 80$ and $300 M_{\odot}$. However, it is only at the highest mass that we expect a major episode of mass loss, since earlier on the interior luminosity always remains slightly below the critical value. Even in this case, however, we anticipate a short lived phase of wind activity related to the small extent of the high opacity surface layer.

Our result of a upper mass limit of $\sim 300 M_{\odot}$ is sensitive to the adopted value of the mass accretion rate and to its time evolution. In fact, $\dot{M}$ may not be constant and decrease in time from an even larger initial value (e.g., Omukai \& Nishi 1998). Quantitatively, it is difficult to predict the exact values of $M_{*}^{\max }$ as a function of $\dot{M}$ without detailed models. However, we can expect that the evolution obtained here will not change qualitatively. In case of larger values of $\dot{M}$, the higher luminosity will cause an earlier stripping of the surface layers by radiation pressure. Also, nuclear burning will be postponed at higher masses because of the shorter contraction time scale. On the contrary, for lower accretion rates, the evolution would resemble that of Pop I protostars with the central core smoothly becoming a ZAMS star during the main accretion phase, but with a fundamental difference. Because of the lack of grains, the radiative force is not capable to reverse the infall until a very large value of the protostellar mass. Thus, it is evident that there must be a critical value of $\dot{M}$ for the onset of the runaway increase of the protostellar radius that determines the maximum accumulated mass. The existence of this critical behavior will be explored in a forthcoming paper.

This work is supported in part by Research Fellowships of the Japan Society for the Promotion of Science for Young Scientists, grant 6819. K.O. wishes to thank the director of the Osservatorio di Arcetri for the hospitality during an extended visit in 2001.

\section{REFERENCES}

Abel, T., Bryan G. L., \& Norman M. L. 2000, ApJ, 540, 39

Bromm, V., Coppi, P. S., \& Larson, R. B. 1999, ApJ, 527, L5 
Bromm, V., Coppi, P. S., \& Larson, R. B. 2001, ApJ, in press (astro-ph/0102503)

Bromm, V., Kudritzki, R. P., \& Loeb, A. 2001, ApJ, 552, 464

Ezer, D., \& Cameron, A. G. W. 1971, Ap\&SS, 14, 399

Iglesias, C. A., \& Rogers, F. J. 1996, ApJ, 464, 943

Larson, R. B., \& Starrfield, S. 1971, A\&A, 13, 190

Lenzuni, P., Chernoff, D. F., \& Salpeter, E. E. 1991, ApJS, 76, 759

Marigo, P., Girardi, L., Chiosi, C., \& Wood, P. 2001, A\&A, 371, 152

Nakano, T. 1989, ApJ, 345, 464

Nakano, T., Hasegawa, T., \& Norman, C. 1995, ApJ, 450, 183

Omukai, K., \& Nishi, R. 1998, ApJ, 508, 141

Palla, F., Salpeter, E. E., \& Stahler, S. W. 1983, ApJ, 271, 632

Stahler, S. W., Palla, F., Salpeter, E. E. 1986, ApJ, 302, 590 (SPS)

Stahler, S. W., Shu, F. H., \& Taam, R. E. 1980, ApJ, 241, 637

Tsuribe, T. 2001 in ASP Conf. Ser. 222, The Physics of Galaxy Formation, ed. M. Umemura, \& H. Susa (San Francisco:ASP), 27

Wolfire, M. G., \& Cassinelli, J. P. 1987, ApJ, 319, 850 


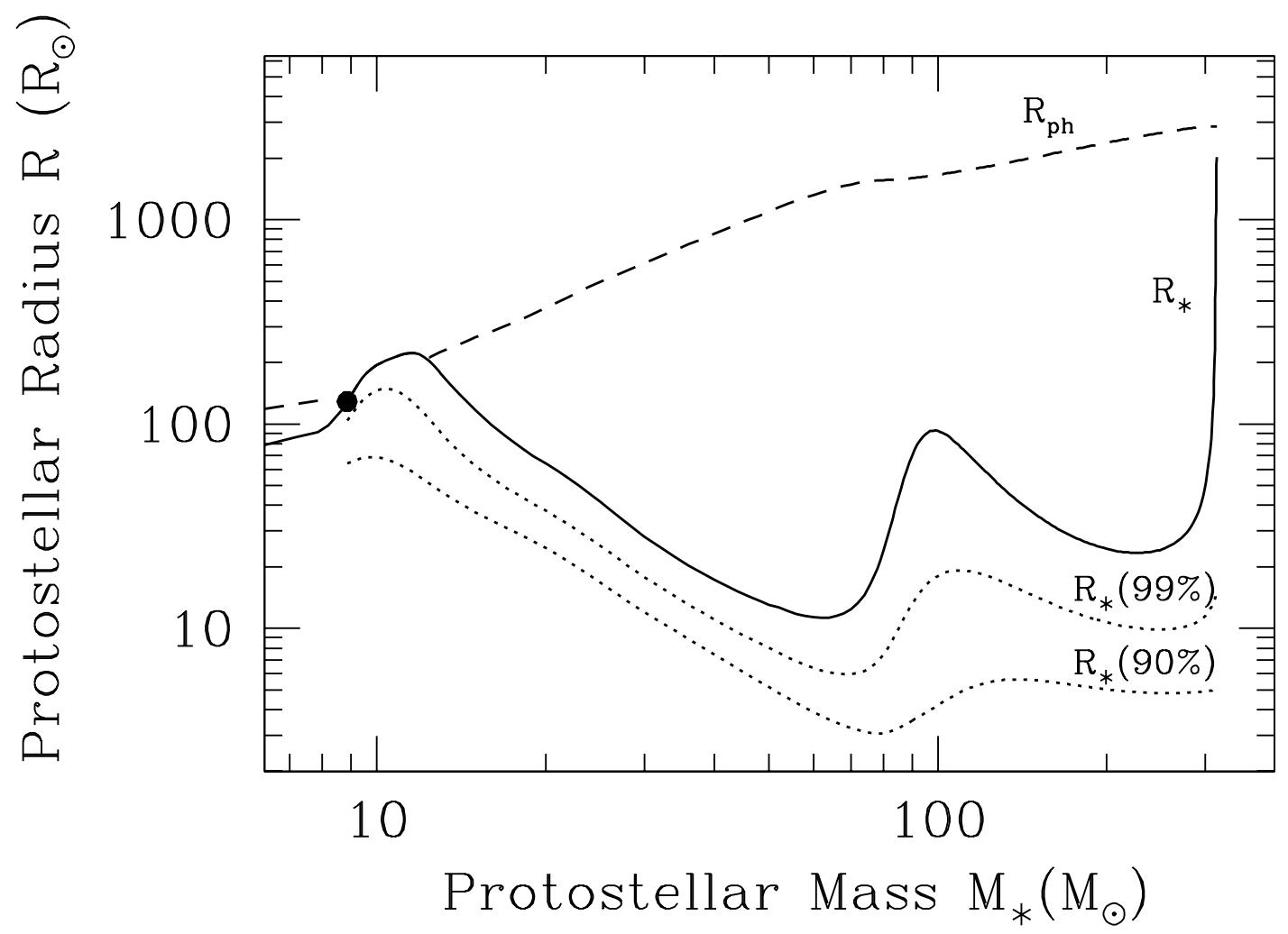

Fig. 1.- Mass-radius relation for massive primordial protostars. The evolution of the core (solid line) and photospheric (dashed line) radii is shown as a function of the protostellar mass. The circle on the curve of $R_{*}$ marks the initial model of our calculations. The $R_{*}$ and $R_{\mathrm{ph}}$ curves at smaller masses come from SPS. The dotted lines represent the radii of mass shells containing $90 \%$ and $99 \%$, respectively, of the total stellar mass. 


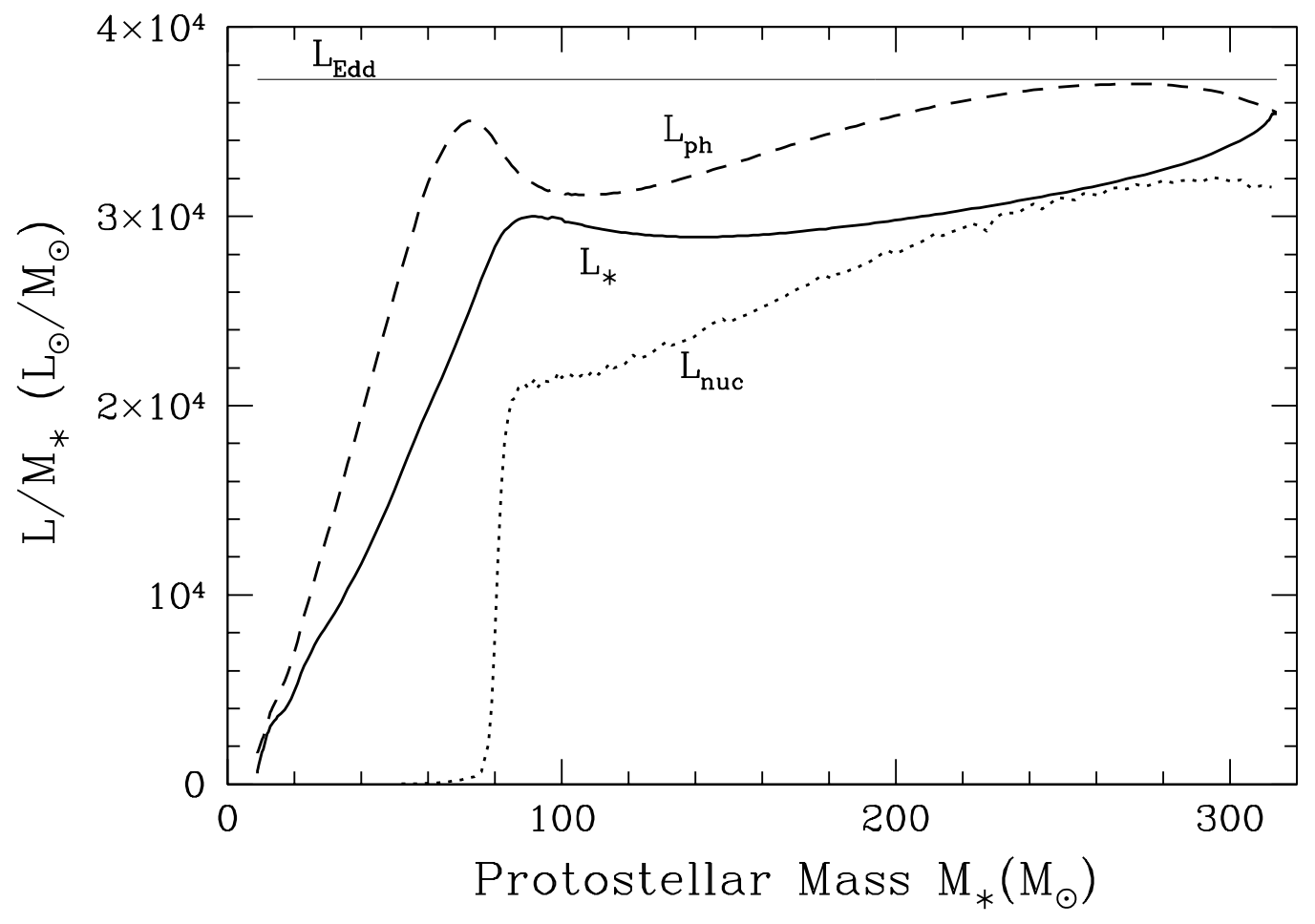

Fig. 2.- Evolution of the luminosity to mass ratio as a function of mass. The interior and photospheric luminosities are displayed by the solid and dotted lines, respectively. The solid horizontal line represents the Eddington luminosity for electron scattering. The nuclear luminosity due to H-burning is shown by the dashed line. 


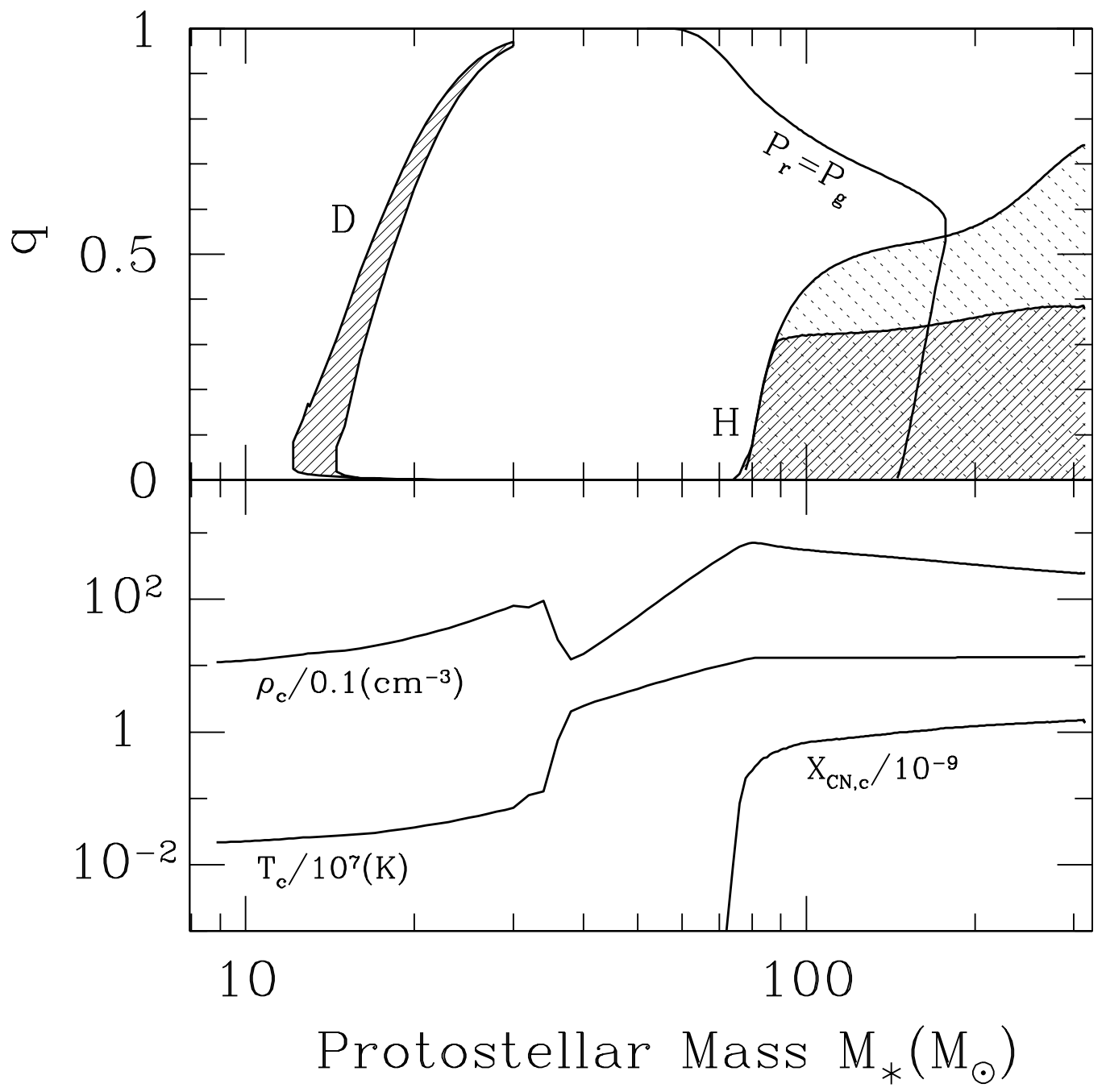

Fig. 3. - The internal structure of the core as a function of mass. (a) Evolution of the nuclear energy and convection as a function of the relative mass $q \equiv M / M_{*}$. Regions where the energy generation by D- and H-burning exceeds $10 \%$ of the average energy generation rate $L_{*} / M_{*}$ are shown as shaded regions The extent of the convective core is illustrated by the short-dashed area. The curve labelled $P_{\mathrm{r}}=P_{\mathrm{g}}$ is the locus where the radiaton pressure equals gas pressure. Radiation pressure dominates to the right of this curve. (b) Evolution of central density, temperature, and mass fraction of CN-elements. Each variable is normalized to $0.1 \mathrm{~g} \mathrm{~cm}^{-3}, 10^{7} \mathrm{~K}$, and $10^{-9}$, respectively. 


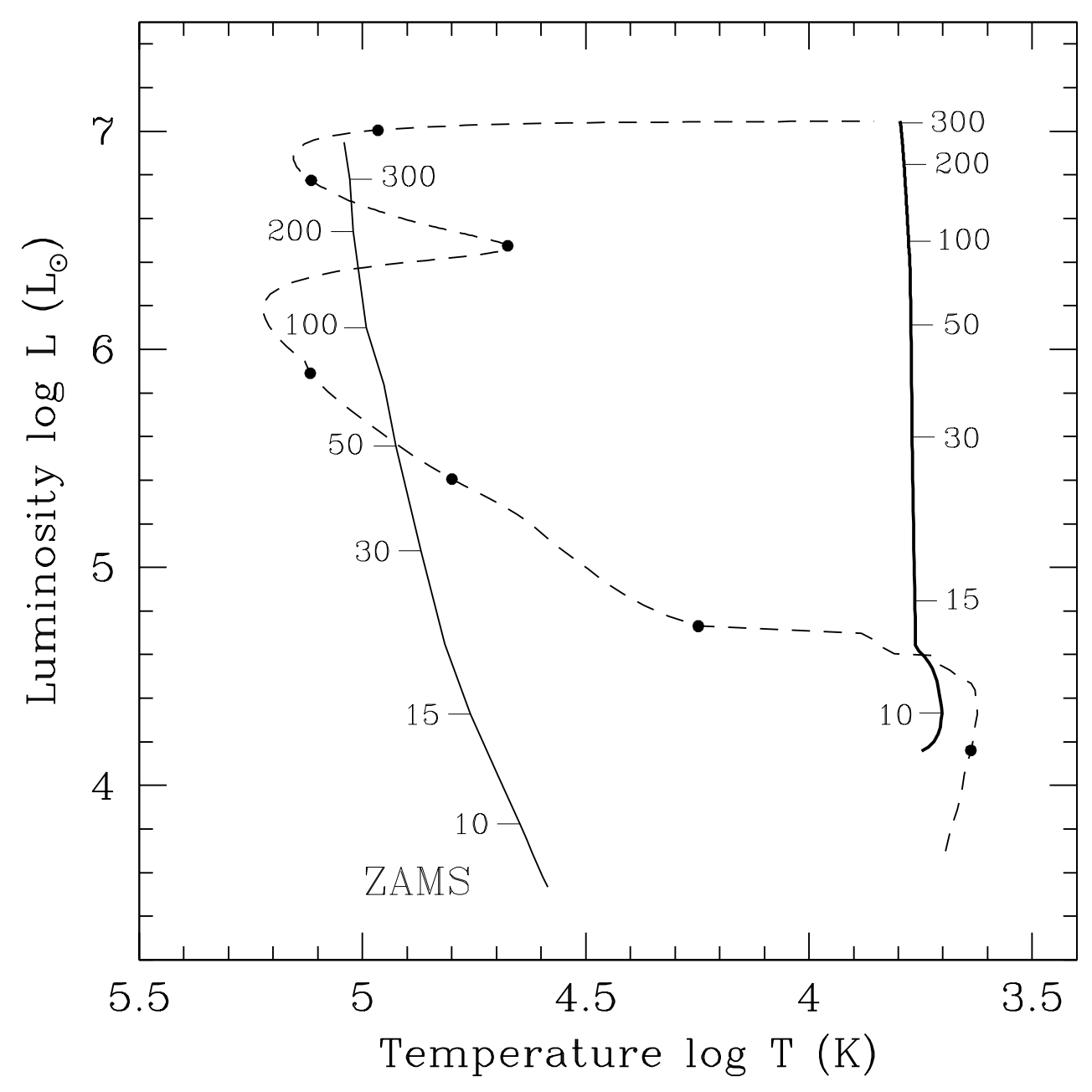

Fig. 4. - HR diagram for primordial protostars. The evolution of the photosphere and core surface is shown by the thick solid and dashed lines, respectively. For comparison, we also show the locus of the metal-free ZAMS stars (Marigo et al. 2001 for $M_{*}<100 M_{\odot}$; Bromm, Kudritzki, \& Loeb 2001 for higher mass). The numbers on both tracks label the value of the core mass (in solar units). The filled circles on the dashed line have the same meaning. 